\section{Contribuições da investigação dos óbitos fetais para melhoria da definição da causa básica do óbito no Município de São Paulo, Brasil}

\section{Contributions by the investigation of fetal deaths for improving the definition of underlying cause of death in the city of São Paulo, Brazil}

\section{Contribuciones de la investigación de óbitos fetales para la mejoría de la definición de la causa básica de fallecimiento en el municipio de São Paulo, Brasil}

\author{
Lays Janaina Prazeres Marques 1 \\ Zilda Pereira da Silva 1 \\ Gizelton Pereira Alencar 1 \\ Marcia Furquim de Almeida 1
}

doi: 10.1590/0102-311X00079120

\title{
Resumo
}

O objetivo desta pesquisa foi analisar a evolução temporal da taxa de mortalidade fetal (TMF) e a contribuição da investigação para a melhoria da definição da causa básica do óbito fetal no Município de São Paulo, Brasil, segundo local de emissão da declaração de óbito. Na abordagem ecológica, analisou-se a tendência da TMF por estrato de peso $(<2.500 \mathrm{~g} e \geq 2.500 \mathrm{~g})$ e óbitos totais no Município de São Paulo entre 2007-2017. Utilizou-se a regressão linear generalizada de Prais-Winsten. No estudo de casos, foram analisadas as causas básicas de óbito fetal de 2012 a 2014, antes e após a investigação, o tempo de conclusão da investigação e a redefinição da causa básica por tipo de atestante. Houve tendência de aumento (1,5\% ao ano) da TMF dos óbitos com $<2.500 \mathrm{~g}$ e de redução $(-1,3 \%$ ao ano) naqueles com $\geq 2.500 \mathrm{~g}$. Os óbitos totais apresentaram tendência estacionária. Entre 2012-2014, cerca de 90\% dos óbitos com $\geq 2.500 \mathrm{~g}$ foram investigados. Após a investigação, houve redefinição da causa básica de morte em 15\% dos casos, e a morte fetal não especificada (P95) representou 25\% das causas de óbito. A proporção mais elevada de alteração da causa de morte ocorreu nos casos cuja Declaração de Óbito foi emitida pelos serviços de verificação de óbito (17\%), ao passo que nos serviços de saúde foi de 10,6\%. Concluiu-se que a TMF dos óbitos com $\geq 2.500 \mathrm{~g}$ apresentou tendência de redução. Houve redefinição significativa das causas básicas, sobretudo naquelas atestadas pelo serviços de verificação de óbito. Entretanto, foi insuficiente para ampliar a proporção de causas de morte que permitissem maior compreensão das condições de mortalidade.

Mortalidade Fetal; Causa Básica de Morte; Estudos de Séries Temporais

\author{
Correspondência \\ L. J. P. Marques \\ Faculdade de Saúde Pública, Universidade de São Paulo. \\ Av. Dr. Arnaldo 715, São Paulo, SP 01246-904, Brasil. \\ laysjpmarques@gmail.com \\ 1 Faculdade de Saúde Pública, Universidade de São Paulo, São \\ Paulo, Brasil.
}




\section{Introdução}

A mortalidade fetal é um dos problemas negligenciados na saúde global atual 1,2. A classificação precisa e consistente das causas e condições associadas aos natimortos é essencial para subsidiar estratégias para reduzir os mais de 2,6 milhões de óbitos fetais que ocorrem a cada ano em todo o mundo 2. Nos últimos anos, foram estabelecidos compromissos para reduzir a mortalidade fetal até 2030, que incluíram o aprimoramento da coleta de dados e a ampliação das pesquisas, bem como o aprofundamento do conhecimento dos mecanismos causais e a articulação de fatores biológicos e epidemiológicos dos óbitos fetais 3 .

A taxa de mortalidade fetal (TMF) configura indicador importante da qualidade da assistência prestada durante a gestação e o parto ${ }^{1}$. No período de 2000 a 2015, a TMF mundial apresentou redução de $25,5 \%$, passando de 24,7 a 18,4 óbitos fetais por mil nascimentos, respectivamente ${ }^{4}$. No Brasil, a TMF variou de 4,9 a 5,8 por mil nascimentos entre 2000 e $2016^{5}$, com tendência estacionária a partir de 2000. O país acompanha o perfil mundial em que os óbitos fetais se constituem no componente predominate da mortalidade perinatal 6 .

Uma questão nuclear no estudo da mortalidade fetal tem sido a dificuldade de determinação das causas de morte 7 . Tradicionalmente, essas causas têm sido empregadas como ferramenta para o conhecimento das condições que favoreceram a ocorrência dos óbitos e para o planejamento de estratégias para sua redução. Em todo mundo, proporção considerável de óbitos permanece com causa indeterminada, principalmente devido à ausência de avaliações pós-morte (autópsia, revisão dos prontuários clínicos e dos atestados de óbito fetal e exames patológicos da placenta) ou, quando realizadas, por serem incompletas e mal interpretadas 8. Diversas iniciativas do governo brasileiro foram desenvolvidas com vistas à melhoria da qualidade dessas informações. Entre elas estão a capacitação de médicos, o fortalecimento dos Serviços de Verificação de Óbito (SVO) e dos Institutos de Medicina Legal (IML), além da estruturação dos comitês de investigação do óbito infantil e fetal 9,10.

A estruturação dos Comitês de Prevenção do Óbito Infantil e Fetal em todo o país ocorreu a partir de meados da década de 1990, com algumas iniciativas estaduais e municipais desenvolvidas a partir de sua realidade, interesses e condições de operacionalização 11. Em 2010, ampliando as ações já executadas pelos comitês de mortalidade, foi instituída a Vigilância dos Óbitos Infantis e Fetais (VOIF) 11. As ações da VOIF está estruturada em quatro etapas: (i) identificação dos óbitos e validação do endereço de residentes no município, (ii) investigação epidemiológica, (iii) discussão do caso e (iv) correção ou complementação dos dados nos sistemas de informações sobre estatísticas vitais com encaminhamento de recomendações para os gestores 12 .

No Estado de São Paulo, o Comitê Estadual de Investigação do Óbito Infantil e Fetal foi implantado em 2006, destinando-se a priorizar a investigação dos óbitos fetais com peso ao nascer considerado como adequado $(\geq 2.500 \mathrm{~g})$ devido ao seu maior potencial de evitabilidade 11. O Município de São Paulo segue a recomendação estadual para investigação. No Município de São Paulo, os óbitos fetais ocorrem em sua quase totalidade nos hospitais e são em sua grande maioria anteparto 13, com a maior parte dos casos encaminhados ao SVO para a determinação da causa de morte via necropsia. Essa rotina teoricamente deveria contribuir para o aprimoramento da indicação da causa básica na capital paulista 14.

Apesar da relevância dos óbitos fetais e das contribuições da investigação para o esclarecimento das circunstâncias da sua ocorrência, são raras as pesquisas que se propuseram a avaliar essa estratégia com enfoque na qualificação dos dados sobre natimortos no país. Assim, destacam-se estudos recentes de análise dos fatores relacionados à não investigação dos óbitos fetal e infantil 15; avaliação da completitude das variáveis das fichas de investigação dos óbitos perinatais 16 e avaliação dos principais indicadores da vigilância do óbito fetal 17.

Nesse sentido, este estudo teve por objetivo analisar a evolução temporal das TMF e a contribuição da investigação na melhoria da definição da causa básica do óbito fetal no Município de São Paulo, segundo local de emissão da declaração de óbito. 


\section{Métodos}

O estudo foi realizado em duas etapas. Na primeira, foi utilizada a abordagem de série temporal para identificar a tendência da mortalidade fetal, considerando dois grupos por peso ao nascer $(<2.500 \mathrm{~g}$ e $\geq 2.500 \mathrm{~g}$ ) e para os óbitos fetais totais. Na segunda, realizou-se um estudo descritivo de casos por meio das causas básicas de morte dos óbitos fetais antes e após a investigação. Foram incluídas as Declarações de Óbito (DO) fetais de mães com residência no Município de São Paulo entre 2007 e 2017. Esse município está localizado na Região Sudeste do Brasil, sendo o mais populoso do país, com cerca de 12 milhões de habitantes, com renda per capita de 4,2 salários mínimos, 50\% de cobertura de planos privados de saúde e baixo nível de analfabetismo (0,13\%) (Instituto Brasileiro de Geografia e Estatística. https://cidades.ibge.gov.br/brasil/sp/sao-paulo/panorama, acessado em 12/Jan/2020).

Utilizaram-se as informações do Sistema de Informações sobre Mortalidade (SIM) e do Sistema de Informações sobre Nascidos Vivos (SINASC) para calcular a TMF por meio do quociente dos óbitos fetais a partir da $22^{2}$ semana de gestação pela soma do número total de nascidos vivos e óbitos fetais 11 .

Para a análise da tendência temporal, foi empregada a regressão linear generalizada de PraisWinsten. A variação percentual anual (em inglês, annual percent change - APC) da TMF foi obtida para os estratos de peso $(<2.500 \mathrm{~g} \mathrm{e} \geq 2.500 \mathrm{~g})$ e para os óbitos totais. O processo de modelagem dos dados incluiu a transformação das TMF em uma função logarítmica de base 10 (variável dependente), e os anos-calendário como variável independente. Os pressupostos de normalidade e homocedasticidade foram verificados pelos testes de Shapiro \& Wilk e Breusch-Pagan-Godfrey, respectivamente. A presença de autocorrelação foi avaliada pela estatística Durbin-Watson, com base na pontuação de 0 a 4, identificando os valores próximos de 2 que significam ausência de autocorrelação serial. Após a modelagem, calculou-se a APC com base na fórmula: $A P C=\left[-1+10^{\beta_{1}}\right] x 100$, e os respectivos intervalos de $95 \%$ de confiança: $I C 95 \%=\left[-1+10^{\beta_{1 \min }}\right] x 100 ;\left[-1+10^{\beta_{\max }}\right] x 100$. Os valores de $\mathrm{p}$ foram obtidos pelo teste de Wald 18, sob o nível de significância de 5\%. Em seguida, foi identificado se o comportamento das taxas era estacionário $(\mathrm{p} \geq 0,05)$, decrescente $(\mathrm{p}<0,05$ e coeficiente de regressão $\beta_{1}$ negativo) ou crescente ( $\mathrm{p}<0,05$ e coeficiente de regressão $\beta_{1}$ positivo).

Os dados sobre mortalidade são disponibilizados eletronicamente com até dois anos de defasagem na página de Internet do SIM (http://sim.saude.gov.br), que divulga as causas de óbito corrigidas, após a investigação. $\mathrm{O}$ acesso às causas básicas de óbito originais, antes da modificação ocasionada ao final da investigação, só é realizado por meio dos microdados, também disponível na página de Internet do Departamento de Informática do SUS (DATASUS; https://datasus.saude.gov.br/). Assim, coletaramse os dados sobre as causas básicas com o máximo de especificação, que inclui o 4o dígito da codificação da Classificação Internacional de Doenças e Problemas Relacionados à Saúde - 10a revisão (CID-10). Além disso, para avaliar se o óbito foi investigado dentro do prazo estabelecido (120 dias) 11, calculou-se o tempo de conclusão da investigação de cada caso por meio das informações sobre a data da investigação e do recebimento no nível central da última atualização do registro.

A análise de casos incluiu todos os óbitos fetais investigados com peso $\geq 2.500 \mathrm{~g}$. Foi selecionado o período de 2012 a 2014, por demonstrar maior proporção de óbitos investigados. O primeiro aspecto analisado se referiu à comparação das causas básicas antes e após a investigação do óbito, mediante a verificação dos códigos da CID-10. Assim, analisou-se o ranking das dez causas principais antes e após a investigação e seus respectivos pares. O teste de McNemar foi utilizado para verificar a significância da mudança da definição das causas de morte entre os pares do ranking e por tipo de atestante, antes e após a investigação. O nível de significância foi estabelecido em $5 \%$.

Para calcular o percentual de mudança para cada causa antes e após a investigação, optou-se pela estratificação por instituição responsável pela emissão da DO. Assim, selecionou-se o campo 52 da seção VII da DO, referente a "Médico que assina atendeu ao falecido? 1 - Sim; 2 - Substituto; 3 - IML; 4 SVO e 5 - Outros". Os campos 1, 2 e 5 foram agrupados em "serviços de saúde (SS)". Os campos 3 e 4 foram agrupados como "Serviço de Verificação de Óbito (SVO)". Verificou-se a independência entre as categorias da variável tipo de serviço atestante (SS/SVO) por meio do teste do qui-quadrado de Pearson $\left(\chi^{2}\right)(\mathrm{p}<0,05)$.

Os dados foram tabulados e analisados por meio do Excel (https://products.office.com/), do Predictive Analytics SoftWare, versão 18.0 (https://www.ibm.com/) e do Stata 13 (https://www.stata. com). Com base na Resolução no 466/12 do Conselho Nacional de Saúde, o projeto da pesquisa foi 
aprovado pelo Comitê de Ética em Pesquisa da Faculdade de Saúde Pública da Universidade de São Paulo (CEP/FSP/USP) (parecer no 3.215.709).

\section{Resultados}

No período entre 2007 e 2017, ocorreram 16.230 óbitos fetais. A TMF variou de 8,2 a 8,7 óbitos por mil nascimentos, com aumento de cerca de 6\%. A maior concentração dos óbitos ocorreu na categoria de peso $<2.500 \mathrm{~g}(80 \%)$. A taxa de mortalidade dos óbitos fetais de baixo peso ao nascer $(<2.500 \mathrm{~g})$ apresentou aumento de $15,8 \%$. Os óbitos com $\geq 2.500$ g representavam 25\% em 2007, passando para 18\% em 2017, correspondendo a uma redução de 15,8\% da TMF no mesmo período (Figura 1).

A análise estratificada por peso demonstrou tendência de aumento da TMF de 1,5\% ao ano nos óbitos com < 2.500g (APC = 1,5\%; IC95\%: 0,39; 2,59) e redução anual de 1,3\% nos óbitos com peso de $\geq 2.500 \mathrm{~g}$ (APC = -1,3\%; IC95\%: -2,30; -0,30). Os modelos para os dois estratos de peso foram estatisticamente significativos $(\mathrm{p}<0,05)$. Entretanto, houve tendência estacionária da TMF dos óbitos fetais totais $(\mathrm{p} \geq 0,05)$. Constatou-se a ausência de autocorrelação serial, indicada pelos valores próximos de 2 para os três modelos (Tabela 1 ).

A investigação no Município de São Paulo se concentrou nos óbitos fetais de $\geq 2.500 \mathrm{~g}$. Verificouse que a investigação dos óbitos só foi efetivamente implantada a partir de 2010. No período anterior, essa prática não correspondia a 10\% do total de óbitos. Entre 2012 e 2014, foram investigados praticamente $90 \%$ dos óbitos. A partir destes resultados, decidiu-se que a comparação das causas de morte antes e depois da investigação iria se concentrar nesse período, pois estaria sendo coberta a maioria dos óbitos (Tabela 2).

Do total de 4.103 óbitos fetais registrados no SIM entre 2012 e 2014, 789 (29,2\%) atendiam ao critério de peso para investigação ( $\geq 2.500)$. Desses, 720 (91,3\%) foram investigados, sendo a maioria, 554 casos (76,9\%), atestada pelo SVO, e o restante, 142 (19,7\%) casos, pelos serviços de saúde. Em 24 $(3,3 \%)$ casos, a variável atestante do óbito teve preenchimento ignorado ou em branco (Figura 2).

Em relação aos óbitos investigados, a quase totalidade foi anteparto $(92,6 \%)$, ocorreu em hospital $(99,6 \%)$ e teve a realização de necropsia $(70,6 \%)$. O tempo de conclusão da investigação esteve dentro do período recomendado em apenas 161 (22,4\%) óbitos. O tempo mínimo de atraso foi de 6 dias e o máximo de 715 dias. Em média, todo o processo investigativo ocorreu em 319 dias. A mediana de investigação foi de 306 dias.

\section{Figura 1}

Taxa de mortalidade fetal (TMF), por mil nascimentos, segundo peso ao nascer. Município de São Paulo, Brasil, 2007-2017.

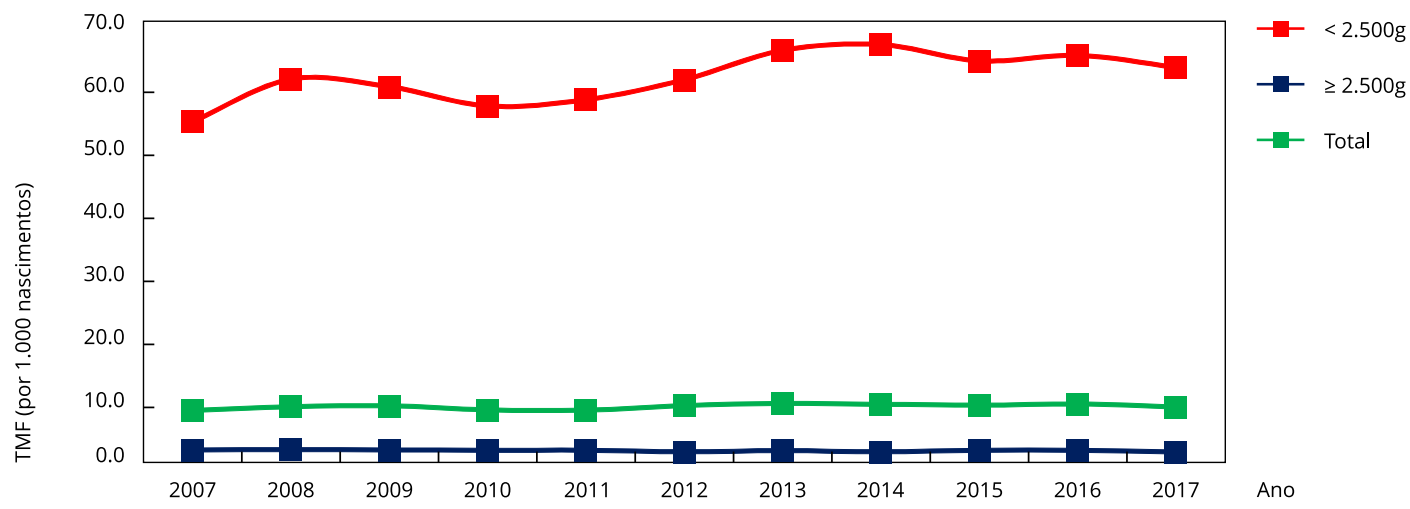

Fonte: Sistema de Informações sobre Mortalidade (SIM) e Sistema de Informações sobre Nascidos Vivos (SINASC). 
Tabela 1

Estimativas da regressão de Prais-Winsten para a taxa de mortalidade fetal (por mil nascimentos) segundo estrato de peso. Munícipio de São Paulo, Brasil, 2007-2017.

\begin{tabular}{lccccc}
\hline Peso ao nascer $(\mathbf{g})$ & $\boldsymbol{\beta}_{\mathbf{1}}$ & Valor de $\mathbf{p}$ * & APC (\%) & IC95\% & Tendência \\
\hline$<2.500$ & 0,0064 & $<0,05$ & 1,5 & 0,$39 ; 2,59$ & Crescente \\
$\geq 2.500$ & $-0,0057$ & $<0,05$ & $-1,3$ & $-2,30 ;-0,30$ & Decrescente \\
Total & 0,0034 & $\geq 0,05$ & 0,8 & $-0,15 ; 1,74$ & Estacionária \\
\hline
\end{tabular}

APC: variação percentual anual (anual percent change); IC95\%: intervalo de 95\% de confiança;

$\beta_{1}$ : coeficiente de regressão.

Fonte: Sistema de Informações sobre Mortalidade (SIM) e Sistema de Informações sobre Nascidos Vivos (SINASC).

* Teste de Wald.

Tabela 2

Percentual de óbitos fetais investigados segundo peso ao nascer. Município de São Paulo, Brasil, 2007-2017.

\begin{tabular}{|c|c|c|c|c|c|c|}
\hline \multirow[t]{3}{*}{ Ano } & \multicolumn{3}{|c|}{$<2.500 \mathrm{~g}$} & \multicolumn{3}{|c|}{$\geq 2.500 \mathrm{~g}$} \\
\hline & \multirow{2}{*}{$\begin{array}{c}\text { Ocorridos } \\
\text { n }\end{array}$} & \multicolumn{2}{|c|}{ Investigados } & \multirow{2}{*}{$\begin{array}{c}\text { Ocorridos } \\
\text { n }\end{array}$} & \multicolumn{2}{|c|}{ Investigados } \\
\hline & & $\mathbf{n}$ & $\%$ & & $\mathbf{n}$ & $\%$ \\
\hline 2007 & 902 & 12 & 1,3 & 296 & 6 & 2,0 \\
\hline 2008 & 1.013 & 6 & 0,6 & 307 & 1 & 0,3 \\
\hline 2009 & 1.024 & 19 & 1,9 & 297 & 24 & 8,1 \\
\hline 2010 & 958 & 104 & 10,9 & 285 & 123 & 43,2 \\
\hline 2011 & 987 & 86 & 8,7 & 291 & 48 & 16,5 \\
\hline 2012 & 1.064 & 99 & 9,3 & 253 & 240 & 94,9 \\
\hline 2013 & 1.115 & 121 & 10,9 & 279 & 254 & 91,0 \\
\hline 2014 & 1.135 & 138 & 12,2 & 257 & 226 & 87,9 \\
\hline 2015 & 1.091 & 39 & 3,6 & 291 & 30 & 10,3 \\
\hline 2016 & 1.062 & 7 & 0,7 & 280 & 1 & 0,4 \\
\hline 2017 & 1.048 & - & - & 238 & - & - \\
\hline
\end{tabular}

Fonte: Sistema de Informações sobre Mortalidade (SIM).

Dos 720 óbitos fetais investigados, 109 (15,1\%) registraram alteração na causa básica de morte. Entretanto, as principais mudanças ocorreram nas posições do ranking. As dez principais causas de morte antes da investigação se referiam a 94\% do total de óbitos e, após a investigação, foram reduzidas para $92 \%$. Verificou-se que as três principais causas de morte permaneceram as mesmas antes e após a investigação, havendo alteração na posição e na representatividade, que passaram de 79,3\% do total de óbitos para 72,9\% após a investigação (Tabela 3).

Os óbitos fetais de causa não especificada (P95) ocupavam o primeiro posto inicialmente e, após a investigação, houve uma retificação significativa de 14,4\%, passando a ocupar o terceiro posto, com a maioria (20\%) da realocação realizada para outras formas de descolamento da placenta e hemorragia (P02.1). A causa básica que apresentou maior percentual de alteração após a investigação foi a hipóxia não especificada (P20.9), com 31\% dos óbitos definidos originalmente, modificados em sua maioria $(25,7 \%)$ para a hipóxia intrauterina diagnosticada antes do início do trabalho de parto (P20.0), que passou a ocupar o primeiro posto após a investigação. As causas por hipóxia intrauterina diagnosticada antes do início do trabalho de parto (P20.0) tiveram modificação de 9,1\%, com os óbitos atribuídos a outras 11 causas. Após a investigação, a síndrome do filho de mãe com diabetes gestacional (P70.0), 
Figura 2

Fluxograma da investigação dos óbitos fetais. Município de São Paulo, Brasil, 2012-2014.

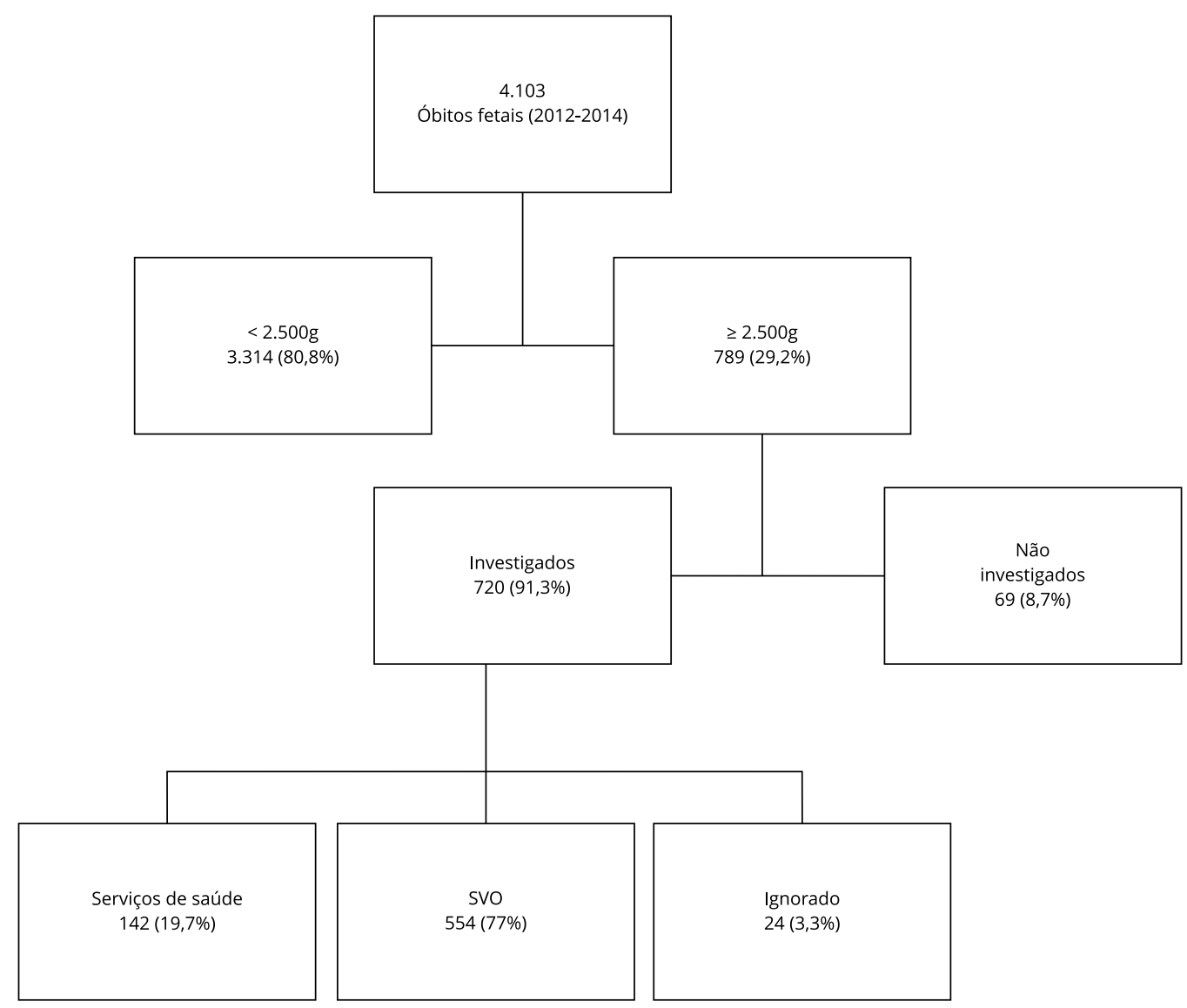

SVO: Serviço de Verificação de Óbito.

Fonte: Sistema de Informações sobre Mortalidade (SIM).

que representou $1 \%$ dos óbitos, ocupou a décima posição, empatada com as doenças infecciosas e parasitárias da mãe (P00.2), que não foram incluídas no ranking antes da investigação, pois ocupavam a 12a posição (Tabela 3 ).

No período de 2012 a 2014, dos 554 (76,9\%) óbitos fetais atestados pelo SVO, houve redefinição das causas básicas em 17\% $(\mathrm{n}=94)$ após a investigação. As três principais causas de morte indicadas pelo SVO apresentaram mudança significativa após a investigação. A morte fetal de causa não especificada (P95) representou a principal causa básica de morte antes da investigação, e cerca de $15 \%$ $(\mathrm{n}=30)$ desses óbitos foram reclassificados para outras 14 causas, sendo o maior número referente às outras formas de descolamento da placenta e hemorragia (P02.1). Após a investigação, o total de óbitos com essa menção $(\mathrm{n}=171)$ se aproximou daqueles representados pela segunda causa de morte após a investigação, a hipóxia intrauterina diagnosticada antes do início do trabalho de parto (P20.0) $(\mathrm{n}=170)$. A hipóxia intrauterina não especificada (P20.9) representou a maioria das reclassificações, com $48(4,8 \%)$ casos, sendo a maior parte das causas classificadas para hipóxia intrauterina diagnosticada antes do início do trabalho de parto (P20.0) (Tabela 4). Houve independência entre as variáveis SVO e serviços de saúde $\left(\chi^{2}=2,85 ; \mathrm{p}<0,05\right)$. 
Principais causas básicas dos óbitos fetais antes e após a investigação. Município de São Paulo, Brasil, 2012-2014.

\begin{tabular}{|c|c|c|c|c|c|c|c|}
\hline \multirow[t]{2}{*}{ Causa básica } & \multicolumn{3}{|c|}{ Antes da investigação } & \multicolumn{3}{|c|}{ Após a investigação } & \multirow[t]{2}{*}{ Valor de $p$ * } \\
\hline & $\mathbf{n}$ & $\%$ & Ranking & $\mathbf{n}$ & $\%$ & Ranking & \\
\hline Morte fetal de causa não especificada (P95) & 208 & 28,9 & $1 \underline{0}$ & 180 & 25,0 & 2으 & $<0,05$ \\
\hline Hipóxia intrauterina não especificada (P20.9) & 198 & 27,5 & 2o & 142 & 19,7 & 3o & $<0,05$ \\
\hline $\begin{array}{l}\text { Hipóxia intrauterina diagnosticada antes do início do trabalho } \\
\text { de parto (P20.0) }\end{array}$ & 165 & 22,9 & 3으 & 203 & 28,2 & 1 으 & $<0,05$ \\
\hline Outras compressões do cordão umbilical (P02.5) & 29 & 4,0 & 4o & 32 & 4,4 & 4으 & $\geq 0,05$ \\
\hline $\begin{array}{l}\text { Outras formas de descolamento da placenta e hemorragia } \\
\text { (P02.1) }\end{array}$ & 23 & 3,2 & 5으 & 30 & 4,2 & 5으 & $<0,05$ \\
\hline $\begin{array}{l}\text { Anormalidades morfológicas e funcionais da placenta e as não } \\
\text { especificadas (P02.2) }\end{array}$ & 16 & 2,2 & 6o & 17 & 2,4 & 7으 & $\geq 0,05$ \\
\hline Transtornos maternos hipertensivos (P00.0) & 11 & 1,5 & 7으 & 18 & 2,5 & 6o & $<0,05$ \\
\hline Malformação não especificada do coração (Q24.9) & 11 & 1,5 & 8으 & 11 & 1,5 & 8으 & $\geq 0,05$ \\
\hline Síndrome do filho de mãe com diabetes gestacional (P70.0) & 7 & 1,0 & 9o & 10 & 1,4 & $10 \underline{0}$ & $\geq 0,05$ \\
\hline Síndrome do filho de mãe diabética (P70.1) & 6 & 0,8 & 10 o & 11 & 1,5 & 9o & $\geq 0,05$ \\
\hline Doenças infecciosas e parasitárias da mãe (P00.2) & 3 & 0,4 & 12 o & 10 & 1,4 & 10 o & $<0,05$ \\
\hline Demais causas & 43 & 6,0 & - & 56 & 7,8 & - & - \\
\hline Total & 720 & 100,0 & - & 720 & 100,0 & - & - \\
\hline
\end{tabular}

Fonte: Sistema de Informações sobre Mortalidade (SIM).

* Teste de McNemar.

Dos 142 (23,1\%) óbitos fetais investigados com DO atestada por médicos dos serviços de saúde, $11 \%(\mathrm{n}=15)$ apresentaram mudança na causa básica original. Apenas as causas de hipóxia intrauterina diagnosticada antes do início do trabalho de parto (P20.0) e de hipóxia intrauterina não especificada (P20.9) apresentaram alteração de 2 (10\%) e 13 óbitos (3,7\%), respectivamente. A maior parte das causas de hipóxia intrauterina não especificada (P20.9) foi reclassificada como hipóxia intrauterina diagnosticada antes do início do trabalho de parto (P20.0) (Tabela 4).

\section{Discussão}

A TMF dos óbitos totais mostrou-se estável entre 2007 e 2017. No entanto, houve aumento da taxa no estrato de peso com $<2.500 \mathrm{~g}$ e redução naqueles com $\geq 2.500 \mathrm{~g}$. Cerca de $90 \%$ dos óbitos com $\geq$ $2.500 \mathrm{~g}$ foram investigados no período de 2012 a 2014. A redefinição da causa básica de morte após a investigação ocorreu em 15\% desses óbitos. Entretanto, mesmo após a investigação, a morte fetal não especificada (P95) ainda correspondia a 25\% das causas. A proporção mais elevada de alteração da causa de morte ocorreu nos óbitos cuja DO foi emitida pelo SVO.

A tendência de estabilidade da TMF no Município de São Paulo é semelhante à apontada em estudo para o Brasil 6. Houve uma flutuação das taxas no período, aumentando de 8,2 por mil nascimentos em 2007 para 9,3 em 2013, e voltando a cair para 8,7 em 2017. Contudo, os valores da TMF estão abaixo da meta proposta pelo Plano de Ação para Cada Recém-Nascidos 2, iniciativa para prevenir a mortalidade fetal e materna, que prevê para 2030 uma TMF igual ou inferior a 12 óbitos fetais por mil nascimentos. Ainda assim, essa taxa é superior àquelas encontradas em países latinos, ao se considerarem os óbitos fetais a partir da 28a semana de gestação, a exemplo de Argentina $(4,6)$, Chile (3,1), Uruguai (6,6), México (5,5), Costa Rica (6,0), que em 2015 já apresentavam TMF inferior às encontradas para 2017 no Município de São Paulo 19.

À semelhança dos países desenvolvidos 20, a maior parte da mortalidade fetal esteve concentrada nos óbitos fetais anteparto e com peso $<2.500 \mathrm{~g}$. Considerando que o peso ao nascer pode ser utilizado 
Tabela 4

Distribuição dos óbitos fetais com $\geq 2.500 \mathrm{~g}$, segundo causa básica atestada pelo Serviço de Verificação de Óbito (SVO) e pelos serviços de saúde, antes e após a investigação. Município de São Paulo, Brasil, 2012-2014.

\begin{tabular}{|c|c|c|c|c|c|c|c|c|c|c|c|c|c|c|c|}
\hline \multirow[t]{2}{*}{$\begin{array}{l}\text { Causa básica } \\
\text { original }\end{array}$} & \multicolumn{11}{|c|}{ Causa básica após a investigação - SVO } & \multicolumn{3}{|c|}{$\begin{array}{l}\text { Retificação após } \\
\text { investigação }\end{array}$} & \multirow[t]{2}{*}{ Valor de $p$ * } \\
\hline & Total & P95 & P20.0 & P20.9 & P02.1 & P00.0 & P02.5 & Q24.9 & P00.2 & P70.1 & P70.0 & $\begin{array}{l}\text { Demais } \\
\text { causas }\end{array}$ & $\mathbf{n}$ & $\%$ & \\
\hline Total & 554 & 171 & 170 & 116 & 17 & 9 & 8 & 7 & 6 & 6 & 5 & 39 & 94 & 17,0 & - \\
\hline P95 & 199 & 169 & 2 & 5 & 6 & 4 & 1 & 1 & 3 & 2 & - & 6 & 30 & 15,1 & $<0,05$ \\
\hline P20.9 & 158 & - & 40 & 110 & 1 & 2 & 1 & - & - & 1 & 1 & 2 & 48 & 4,8 & $<0,05$ \\
\hline P20.0 & 139 & - & 128 & 1 & - & - & 1 & - & 2 & 1 & 1 & 5 & 11 & 8,0 & $<0,05$ \\
\hline P02.1 & 10 & - & - & - & 10 & - & - & - & - & - & - & - & - & - & $\geq 0,05$ \\
\hline Q24.9 & 7 & - & - & - & - & - & - & 6 & - & - & - & 1 & 1 & 14,2 & $\geq 0,05$ \\
\hline P02.5 & 5 & - & - & - & - & - & 5 & - & - & - & - & - & - & - & $\geq 0,05$ \\
\hline P00.0 & 3 & - & - & - & - & 3 & - & - & - & - & - & - & - & - & $<0,05$ \\
\hline P01.5 & 3 & - & - & - & - & - & - & - & - & - & - & 3 & - & - & $\geq 0,05$ \\
\hline P70.0 & 3 & - & - & - & - & - & - & - & - & - & 3 & - & - & - & $\geq 0,05$ \\
\hline Q89.7 & 3 & - & - & - & - & - & - & - & - & - & - & 3 & - & - & $\geq 0,05$ \\
\hline Demais causas & 24 & 2 & - & - & - & - & - & - & 1 & 2 & - & 19 & 4 & 16,7 & - \\
\hline \multirow[t]{2}{*}{$\begin{array}{l}\text { Causa básica } \\
\text { original }\end{array}$} & \multicolumn{11}{|c|}{ Causa básica após a investigação - serviços de saúde } & \multicolumn{3}{|c|}{$\begin{array}{c}\text { Retificação após } \\
\text { investigação }\end{array}$} & Valor de $p$ * \\
\hline & Total & P20.0 & P20.9 & P02.5 & P02.2 & P02.1 & P95 & P00.0 & Q24.9 & P70.1 & P70.0 & $\begin{array}{l}\text { Demais } \\
\text { causas }\end{array}$ & $\mathbf{n}$ & $\%$ & \\
\hline Total & 142 & 29 & 22 & 19 & 13 & 12 & 9 & 7 & 4 & 4 & 3 & 20 & 15 & 10,6 & - \\
\hline P20.9 & 35 & 11 & 22 & - & - & - & - & - & - & - & - & 2 & 13 & 3,7 & $<0,05$ \\
\hline P20.0 & 20 & 18 & - & - & - & - & - & - & - & 1 & - & 1 & 2 & 10,0 & $<0,05$ \\
\hline P02.5 & 19 & - & - & 19 & - & - & - & - & - & - & - & - & - & - & $\geq 0,05$ \\
\hline P02.2 & 13 & - & - & - & 13 & - & - & - & - & - & - & - & - & - & $\geq 0,05$ \\
\hline P02.1 & 12 & - & - & - & - & 12 & - & - & - & - & - & - & - & - & $\geq 0,05$ \\
\hline P95 & 9 & - & - & - & - & - & 9 & - & - & - & - & - & - & - & $\geq 0,05$ \\
\hline P00.0 & 7 & - & - & - & - & - & - & 7 & - & - & - & - & - & - & $\geq 0,05$ \\
\hline Q24.9 & 4 & - & - & - & - & - & - & - & 4 & - & - & - & - & - & $\geq 0,05$ \\
\hline P70.0 & 3 & - & - & - & - & - & - & - & - & - & 3 & - & - & - & $\geq 0,05$ \\
\hline P70.1 & 3 & - & - & - & - & - & - & - & - & 3 & - & - & - & - & $\geq 0,05$ \\
\hline Demais causas & 17 & - & - & - & - & - & - & - & - & - & - & 17 & - & - & - \\
\hline
\end{tabular}

P00.0: Transtornos maternos hipertensivos; P00.2: Doenças infecciosas e parasitárias da mãe; P01.5: Gravidez múltipla; P02.1: Outras formas de descolamento da placenta e hemorragia; P02.2: Outras anormalidades morfológicas e funcionais da placenta e as não especificadas; P02.5: Outras compressões do cordão umbilical; P20.0: Hipóxia intrauterina diagnosticada antes do início do trabalho de parto; P20.9: Hipóxia intrauterina não especificada; P70.0: Síndrome do filho de mãe com diabetes gestacional; P70.1: Síndrome do filho de mãe diabética; P95: Morte fetal de causa não especificada; Q24.9: Malformação não especificada do coração; Q89.7: Malformações congênitas múltiplas, não classificadas em outra parte.

* Teste de McNemar.

como uma proxy da idade gestacional, pode-se supor que esses casos são provenientes de gestações pré-termo. Resultado semelhante foi apontado por pesquisa realizada no Estado de São Paulo, na qual foi identificado que cerca de $80 \%$ dos óbitos fetais eram provenientes de gestações pré-termo ${ }^{13}$. Os achados do presente estudo demonstraram tendência significativa de declínio nos óbitos de $\geq 2.500 \mathrm{~g}$, considerados como evitáveis. Tendo em vista que esses óbitos são foco das práticas de vigilância em todo o país, dado o seu potencial de evitabilidade, a efetividade das ações de investigação pode ter contribuído para redução dessas mortes, colaborando junto a outros fatores, como o aumento do grau de instrução das mulheres, ampliação da cobertura da atenção primária e hospitalar, aumento 
da quantidade de consultas de pré-natal, disseminação de tecnologias médicas especializadas e maior acesso aos serviços de saúde 21,22.

Em relação à investigação dos óbitos com $\geq 2.500 \mathrm{~g}$, observou-se aumento relevante da proporção de casos investigados após a instituição da obrigatoriedade da vigilância dos óbitos fetais em 2010. Por outro lado, após 2014, houve uma redução da proporção de casos investigados. Tal fato pode estar relacionado à presença de alguns obstáculos para a investigação, tais como: a não localização do endereço da mãe, que impossibilita a validação dos óbitos de residentes no município, pré-requisito para o início da investigação; falta de suporte financeiro; escassez de recursos humanos; preenchimento incompleto dos dados hospitalares; falta de capacitação da equipe; atraso ou ausência da conclusão da investigação e não consolidação dos dados no SIM-Web; assim como a possibilidade de a investigação do óbito fetal ter deixado de ser prioridade institucional no município 12,15,17.

No que se refere ao tempo de conclusão da investigação, a maior proporção dos óbitos foi investigada fora do prazo, e o tempo máximo de atraso foi seis vezes maior que os 120 dias estabelecidos para investigação. Estudo semelhante 17, realizado na Região Nordeste do Brasil, constatou resultados opostos, em que a maioria dos óbitos fetais foi investigada no prazo, e o tempo mínimo de atraso foi de dois dias e o máximo de 393 dias. Os motivos de atrasos no prazo de conclusão da investigação eram, em grande parte, os mesmos que foram apontados para a sua não realização, acrescidos pela rotatividade dos profissionais da equipe de investigação e da falta de encaminhamento dos casos ocorridos fora do município 15,17 .

Observou-se que a maioria das causas básicas mostrou modificação significativa no total de óbitos. No entanto, as causas permaneceram praticamente as mesmas, com alteração apenas no posto ocupado no ranking, à exceção da incorporação dos óbitos por doenças infecciosas e parasitárias da mãe (P00.2) no 10o posto. Ressalta-se que as três principais causas básicas de morte permaneceram as mesmas após a investigação, havendo uma redução dos óbitos classificados nessas causas, o que provocou mudanças no ranking. A morte fetal de causa não especificada (P95) esteve no topo do ranking, tanto antes como após a investigação. Essa causa continuou com elevada proporção de óbitos, que podem praticamente ser considerados como mal definidos, semelhantes aos garbage code, sendo inúteis para a formulação de políticas públicas 23 .

Após a investigação dos óbitos, a hipóxia intrauterina diagnosticada antes do trabalho de parto (P20.0) representou a principal causa de morte. Ainda que essa causa não contribua para identificação da cadeia causal que levou ao óbito, a sua reclassificação é mais coerente com o perfil dos óbitos fetais, que são em sua grande maioria anteparto. Sabe-se que a hipóxia placentária é um mecanismo básico envolvido em diferentes distúrbios da gravidez, que resultam em pré-eclâmpsia e hipertensão materna 24. A baixa oxigenação pode ser provocada por diversos fatores, incluindo a exposição à poluição do ar, altitude elevada e condições maternas, como anemia, infecções, deficiências nutricionais ou obesidade 24,25 .

As causas atribuídas à morte fetal de causa não especificada (P95) somadas à hipóxia intrauterina não especificada (P20.9) representam quase metade de todas as causas após a investigação. Essas causas não contribuem para a identificação das reais circunstâncias da mortalidade 26 . Esse resultado é semelhante ao encontrado em países de baixa e média renda, onde a causa de até metade de todos os natimortos ainda é desconhecida 27 . Observa-se também que uma parcela considerável dos óbitos fetais nos países desenvolvidos é considerada como não explicada, podendo atingir até cerca de um terço dos óbitos 28 .

Mais de 2/3 dos óbitos fetais teve a causa de morte indicada pelo SVO. Esse serviço responde pela maioria dos óbitos do ranking de causas de morte antes da investigação, semelhante a outros achados para o Município de São Paulo 14. Após a investigação, verificou-se uma mudança significativa das causas em grande parte dos óbitos, especialmente na morte fetal não especificada (P95), sendo esta redefinida entre diversas causas. Houve praticamente um empate entre essa causa de morte e a segunda, hipóxia intrauterina diagnosticada antes do início do trabalho de parto (P20.0). Entretanto, a investigação foi insuficiente para ampliar a proporção de causas de morte que possibilitasse uma maior compreensão da cadeia causal dos óbitos. Tal fato é semelhante ao encontrado por estudo realizado no Município de São Paulo, que constatou a falta de acesso ou de informações clínicas da gestação e do parto, obtidas por meio dos prontuários hospitalares 14 como dificuldades na definição da causa básica dos óbitos fetais. 
No que se refere à realização das autopsias no Município de São Paulo, é necessário considerar que as informações dos prontuários das gestantes, assim como as placentas, não são encaminhadas juntamente com os fetos para o SVO, o que seria essencial para a definição mais precisa das causas. Além disso, a maior parte dos óbitos ocorre antes do parto e muitos fetos se encontram macerados, dificultando a identificação da causa de morte 14. Reconhece-se a autopsia fetal e o exame histopatológico da placenta como os testes diagnósticos mais úteis para a avaliação de possíveis causas de natimortos 7.

Em relação às causas básicas atestadas por médicos dos serviços de saúde, a proporção de mudanças de indicação das causas de morte após investigação foi significativamente menor que aquela registrada no SVO. Houve uma melhor identificação da hipóxia intrauterina diagnosticada antes do início do trabalho de parto (P20.0) em relação à indicação antes da investigação, que apontava boa parte desses óbitos como sendo hipóxia intrauterina não especificada (P20.9). Esta mudança condiz com a elevada proporção de óbitos anteparto encontrada neste estudo. Nas demais causas de morte, não houve retificação após a investigação, indicando que as informações registradas nos prontuários hospitalares contribuíram para uma melhor indicação das causas básicas de morte 14 .

Embora a identificação das causas da morte seja fundamental para adotar medidas de prevenção, até recentemente não existia um sistema de classificação passível de utilização para todos os países. Ao invés disso, diversos sistemas foram desenvolvidos ao longo de décadas 29 . Estudo pioneiro realizou uma revisão sistemática dos 81 sistemas de classificação de causas de óbito fetal desenvolvidos globalmente. Foi analisado o alinhamento dos sistemas com os princípios gerais da CID-10, constatando a sua utilização limitada para a classificação de natimortos, com apenas $21 \%$ dos sistemas usando códigos da CID-10. Identificou-se que a maioria dos sistemas foi aplicado apenas nas regiões em que foram desenvolvidos, tornando incompatíveis os dados produzidos e dificultando a compreensão da dimensão de causas específicas de mortes perinatais no mundo 30.

Em reconhecimento à necessidade de classificar com precisão as causas dessas mortes, a Organização Mundial da Saúde (OMS) lançou em 2016 uma nova abordagem para a classificação de mortes perinatais para uso mundial, a "Aplicação da CID-10 da OMS às mortes perinatais" (CID-Mortalidade Perinatal ou CID-PM) ${ }^{30}$. Esse sistema globalmente aplicável exige o registro do tempo das mortes (intraparto e anteparto) e as condições maternas que contribuíram para a morte perinatal. Dessa forma, o binômio mãe-bebê é reconhecido, e as áreas passíveis de intervenções benéficas, tanto maternas como da criança, são identificadas ${ }^{31}$. Apesar da relevância, a CID-PM não é amplamente utilizada, ainda que a sua aplicação se constitua um passo promissor na melhoria da notificação das causas de óbito fetal 32 .

Quanto às limitações inerentes ao estudo, esta é uma pesquisa com dados secundários que pode conter informações incompletas ou erros de registros. Embora com restrições, estes dados são úteis para a avaliação da tendência da TMF. Ressalta-se que os dados provenientes de sistemas de informações oficiais apresentam defasagem em seu tempo de divulgação, e há um atraso na disponibilização dos dados dos óbitos investigados, possivelmente devido às dificuldades encontradas no processo de investigação. Contudo, os dados disponíveis mostraram-se adequados para verificar as mudanças de definição das causas básicas de morte após a investigação.

A TMF com $\geq 2.500 \mathrm{~g}$, alvo das ações de vigilância, apresentou tendência de redução. Houve mudança significativa na maioria das causas básicas de óbito, com maior redefinição naquelas atestadas pelo SVO. Apesar de a investigação do óbito ter contribuído para melhoria da redefinição da causa básica, as mudanças foram insuficientes para a indicação de causas que melhor retratassem os processos fisiopatológicos que resultaram nos óbitos fetais. A existência de muitas classificações para identificar as causas de morte fetal ao redor do mundo sugere que a CID-10 tem limitações na identificação das causas desse tipo de morte. A utilização da CID-PM poderia contribuir para a melhoria da definição das causas básicas de óbito fetal e para o direcionamento das ações em saúde para o binômio mãe-filho 


\section{Colaboradores}

L. J. P. Marques e M. F. Almeida contribuíram para a concepção e desenho do estudo, análise e interpretação dos dados, redação do artigo, revisão crítica do artigo final e aprovação da versão final a ser publicada. Z. P. Silva contribuiu com a concepção e desenho do estudo, análise e interpretação dos dados, revisão crítica do artigo final e aprovação da versão final a ser publicada. G. P. Alencar contribuiu com a revisão crítica do artigo final e a aprovação da versão final a ser publicada.

\section{Informações adicionais}

ORCID: Lays Janaina Prazeres Marques (00000003-4511-4995); Zilda Pereira da Silva ( 00000003-4648-113X); Gizelton Pereira Alencar (00000002-2354-9050); Marcia Furquim de Almeida (0000-0003-0052-1888).

\section{Referências}

1. Bernis L, Kinney MV, Stones W, Hoope-Bender PT, Vivio D, Leisher SH, et al. Stillbirths: ending preventable deaths by 2030 . Lancet 2016; 387:703-16.

2. Blencowe H, Cousens S, Jassir FB, Say L, Chou D, Mathers C, et al. National, regional, and worldwide estimates of stillbirth rates in 2015, with trends from 2000: a systematic analysis. Lancet Glob Health 2016; 4:e98-e108.

3. Flenady V, Gordon A, Bauman A. Stillbirth prevention: the challenges of public campaigns. BJOG 2017; 125:253.

4. Flenady V, Wojcieszek AM, Middleton P, Ellwood D, Erwich JJ, Michael Coory M, et al. Stillbirths: recall to action in high-income countries. Lancet 2016; 387:691-702.

5. GBD 2016 Mortality Collaborators. Global, regional, and national under-5 mortality, adult mortality, age-specific mortality, and life expectancy, 1970-2016: a systematic analysis for the Global Burden of Disease Study 2016. Lancet 2017; 390:1084-150.

6. Barros PS, Aquino EC, Souza MR. Mortalidade fetal e os desafios para a atenção à saúde da mulher no Brasil. Rev Saúde Pública 2019; 53:12.

7. Page JM, Christiansen-Lindquist L, Thorsten V, Parker CB, Reddy UM, Dudley DJ, et al. Diagnostic tests for evaluation of stillbirth: results from the stillbirth collaborative research network. Obstet Gynecol 2017; 129:699-706.

8. Pekkola M, Tikkanen M, Loukovaara M, Lohi J, Paavonen J, Stefanovic V. Postmortem examination protocol and systematic re-evaluation reduce the proportion of unexplained stillbirths. J Perinat Med 2020; 48:771-7.

9. Azevedo BAS, Vanderlei LCM, Mello RJV, Frias PG. Avaliação da implantação dos serviços de verificação de óbito em Pernambuco, 2012: estudo de casos múltiplos. Epidemiol Serv Saúde 2016; 25:595-606.

10. Marques LJP, Pimentel DR, Oliveira CM, Vilela MBR, Frias PG, Bonfim CV. Concordância da causa básica e da evitabilidade dos óbitos infantis antes e após a investigação no Recife, Pernambuco, 2014. Epidemiol Serv Saúde 2018; 27:e20170557.

11. Ministério da Saúde. Manual de vigilância do óbito infantil e fetal e do Comitê de Prevenção do Óbito Infantil e Fetal. 2a Ed. http://bvsms. saude.gov.br/bvs/publicações/manual_obi to_infantil_fetal_2ed.pdf (acessado em 12/ Jan/2020).

12. Oliveira CM, Bonfim CV, Guimarães MJB, Frias PG, Antonino VCS, Medeiros ZM. Vigilância do óbito infantil no Recife, Pernambuco: operacionalização, potencialidades e limites. Epidemiol Serv Saúde 2017; 26:413-9.

13. Alencar GP, Silva ZP, Santos PC, Raspantini $\mathrm{PB}$, Moura BLA, Almeida MF, et al. What is the impact of interventions that prevent fetal mortality on the increase of preterm live births in the State of Sao Paulo, Brazil? BMC Pregnancy Childbirth 2015; 15:152. 
14. Almeida MF, Alencar GP, Schoeps D, Minuci EG, Silva ZP, Ortiz LP, et al. Qualidade das informações registradas nas declarações de óbito fetal em São Paulo, SP. Rev Saúde Pública 2011; 45:845-53.

15. Dutra IR, Andrade GN, Rezende EM, Gazzinelli A. Investigação dos óbitos infantil e fetal no Vale do Jequitinhonha, Minas Gerais, Brasil. REME Rev Min Enferm 2015; 19:605-11.

16. Heráclio IL, Silva MA, Vilela MBR, Oliveira CM, Frias PG, Bonfim CV. Epidemiological investigation of perinatal deaths in RecifePernambuco: a quality assessment. Rev Bras Enferm 2018; 71:2519-26.

17. Maria LFBS, Araujo TVB. Um olhar sobre a vigilância dos óbitos fetais do Jaboatão dos Guararapes, Pernambuco, Brasil, em 2014. Ciênc Saúde Colet 2017; 22:3415-28.

18. Antunes JLF, Cardoso MRA. Uso da análise de séries temporais em estudos epidemiológicos. Epidemiol Serv Saúde 2015; 24:565-76.

19. Pingray V, Althabe F, Vazquez P, Correa M, Pajuelo M, Belizán JM. Stillbirth rates in 20 countries of Latin America: an ecological study. BJOG 2018; 125:1263-70.

20. Lawn JE, Blencowe H, Waiswa P, Amouzou A, Mathers C, Hogan D, et al. Stillbirths: rates, risk factors, and acceleration towards 2030. Lancet 2016; 387:587-603.

21. Zeitlin J, Mortensen L, Cuttini M, Lack N, Nijhuis J, Haidinger G, et al. Declines in stillbirth and neonatal mortality rates in Europe between 2004 and 2010: results from the Euro-Peristat project. J Epidemiol Community Health 2016; 70:609-15.

22. Vanderlei LCM, Frias PG. Advances and challenges in maternal and child health in Brazil. Rev Bras Saúde Matern Infant 2015; 15:157-8.

23. Lima RB, Frederes A, Marinho MF, Cunha CC, Adair T, França EB. Investigation of garbage code deaths to improve the quality of causeof-death in Brazil: results from a pilot study. Rev Bras Epidemiol 2019; 22 Suppl 3:e190004. supl.3.
24. Thompson LP, Pence L, Pinkas G, Song H, Telugu BP. Placental hypoxia during early pregnancy causes maternal hypertension and placental insufficiency in the hypoxic guinea pig model. Biol Reprod 2016; 95:128.

25. Fajersztajn L, Veras MM. Hypoxia: from placental development to fetal programming. Birth Defects Res 2017; 109:1377-85.

26. Barbeiro FMS, Fonseca SC, Tauffer MG, Ferreira MSS, Silva FP, Ventura PM, et al. Óbitos fetais no Brasil: revisão sistemática. Rev Saúde Pública 2015; 49:22.

27. Aminu M, Broek NVD. Stillbirth in low- and middle-income countries: addressing the 'silent epidemic'. Int Health 2019; 11:237-9.

28. Reinebrant HE, Leisher SH, Coory M, Henry S, Wojcieszek AM, Gardener G, et al. Making stillbirths visible: a systematic review of globally reported causes of stillbirth. BJOG 2018; 125:212-24.

29. Leisher SH, Teoh Z, Reinebrant H, Allanson E, Blencowe H, Erwich JJ, et al. Seeking order amidst chaos: a systematic review of classification systems for causes of stillbirth and neonatal death, 2009-2014. BMC Pregnancy Childbirth 2016; 16:295.

30. Leisher SH, Teoh Z, Reinebrant H, Allanson E, Blencowe H, Erwich JJ, et al. Classification systems for causes of stillbirth and neonatal death, 2009-2014: an assessment of alignment with characteristics for an effective global system. BMC Pregnancy Childbirth 2016; 16:269.

31. Allanson E, Tunçalp Ö, Gardosi J, Pattinson RC, Erwich JJHM, Flenady VJ, et al. Classifying the causes of perinatal death: ICD-PM. Bull World Health Organ 2016; 94:79-79A.

32. Allanson ER, Tunçalp Ö, Gardosi J, Pattinson RC, Vogel JP, Erwich J, et al. Giving a voice to millions: developing the WHO application of ICD-10 to deaths during the perinatal period: ICD-PM. BJOG 2016; 123:1896-9. 


\section{Abstract}

This study aimed to analyze the time trend in stillbirth rate $(S B R)$ and the contribution by investigation to improving the definition of underlying cause of stillbirth in the city of São Paulo, Brazil, according to the place where the death certificate was issued. An ecological approach was used to analyze the trend in SBR by weight stratum $(<2,500 \mathrm{~g}$ and $\geq 2,500 \mathrm{~g})$ and total deaths in the city in 2007-2017. Prais-Winsten generalized linear regression was used. The study of cases analyzed the underlying causes of stillbirth from 2012 to 2014, before and after the investigation, time of conclusion of the investigation, and redefinition of the underlying cause of stillbirths by type of issuer. In deaths with $<2,500 \mathrm{~g}$, there was an upward trend in SBR of $1.5 \%$ per year and a reduction $(-1.3 \%$ per year) in stillbirths $\geq 2,500 \mathrm{~g}$. Total deaths presented a stable trend. In 2012-2014, 90\% of deaths with $\geq 2,500 \mathrm{~g}$ were investigated. After investigation, the underlying cause of death was redefined in 15\% of the deaths, and not otherwise specified stillbirth (P95) represented 25\% of the causes of death. The highest proportion of changes in the underlying cause of death occurred in deaths for which the death certificate was issued by the death certification review service (17\%), while in health services the proportion was $10.6 \%$. In conclusion, the SBR in deaths with $\geq 2,500 \mathrm{~g}$ showed a downward trend. There was a significant redefinition of underlying causes, especially in those attested by the death certification review service. However, the redefinition was insufficient to expand the proportion of causes of death that would allow a better understanding of the mortality conditions.

Fetal Mortality; Underlying Cause of Death; Time Series Studies

\section{Resumen}

El objetivo de esta investigación fue analizar la evolución temporal de la tasa de mortalidad fetal (TMF) y la contribución de su investigación para la mejora de la definición de la causa básica del óbito fetal en el municipio de São Paulo, Brasil, según el lugar de emisión de la declaración de defunción. Desde una perspectiva ecológica, se analizó la tendencia de la TMF por estrato de peso $(<2.500 \mathrm{~g} y \geq 2.500 \mathrm{~g})$ y fallecimientos totales en el municipio de São Paulo entre 2007-2017. Se utilizó la regresión lineal generalizada de PraisWinsten. En el estudio de casos se analizaron las causas básicas de óbito fetal de 2012 a 2014, antes y después de la investigación, el tiempo de conclusión de la investigación y la redefinición de la causa básica de los óbitos fetales por tipo de certificado. En las defunciones con $<2.500 \mathrm{~g}$, hubo una tendencia de aumento de la TMF de 1,5\% al año $y$ de reducción ( $-1,3 \%$ al año) en los óbitos fetales con $\geq 2.500 \mathrm{~g}$. Los óbitos totales presentaron tendencia estable. Entre 2012-2014, se investigaron cerca de un 90\% de los óbitos con $\geq 2.500 \mathrm{~g}$. Tras la investigación, se produjo una redefinición de la causa básica de muerte en un $15 \%$ de los óbitos y la muerte fetal no especificada (P95) representó un 25\% de las causas de óbito. La proporción más elevada de alteración de la causa de muerte se produjo en los óbitos, cuya declaración de fallecimiento se emitió por parte del servicio de verificación de óbito (17\%), mientras que en los servicios de salud fue de un 10,6\%. Se concluye que la TMF de los óbitos con $\geq 2.500$ g presentó una tendencia decreciente. Hubo una redefinición significativa de las causas básicas, sobre todo en aquellas certificadas por el servicio de verificación de óbito. No obstante, esta fue insuficiente para ampliar la proporción de causas de muerte que permitiesen una mayor comprensión de las condiciones de mortalidad.

Mortalidad Fetal; Causa Básica de Muerte; Estudios de Series Temporales
Recebido em 18/Abr/2020

Versão final reapresentada em 20/Jul/2020

Aprovado em 28/Jul/2020 